\title{
Mobile Application for Increasing Contextual and Emotional Work Group Awareness
}

\author{
Mikko Salminen ${ }^{1}$, Kari Kallinen ${ }^{1}$, Kliment Yanev ${ }^{1}$, Niklas Ravaja ${ }^{1}$, \\ and Timo Saari ${ }^{1,2}$ \\ ${ }^{1}$ Helsinki School of Economics, Center for Knowledge and Innovation Research, \\ Helsinki, Finland \\ ${ }^{2}$ Temple University, Philadelphia, PA, USA \\ \{Mikko.Salminen, Kari.Kallinen, Kliment.Yanev, \\ Niklas.Ravaja, Timo.Saari\}@hse.fi
}

\begin{abstract}
This paper presents a prototype of a mobile application enhancing emotional and contextual awareness in distributed knowledge work teams. Emotional, contextual, and other types of data from users are collected both implicitly and explicitly. The benefits of such a system are hypothesized to include increased individual and group awareness, emotional awareness, location- , task- and status awareness. A novel way to visualize data on location, use context, and user's subjective emotional state on a mobile phone are also presented.
\end{abstract}

Keywords: Mobile social application, knowledge work, contextual awareness, emotional awareness.

\section{Awareness in Groups}

In many group work situations, awareness of others provides information that is critical for smooth and effective collaboration. Although group awareness is taken for granted in face-to-face work, it is difficult to maintain in distributed settings. Studies of distributed work have shown that much of the communication and implicit information that is available to a co-located team does not exist for remote collaborators. For example, Herbsleb and Grinter [1] found that lack of ad-hoc communication between software developers caused an increase in coordination problems and a decrease in collaboration between remote sites.

We define group emotion as the group's affective state that arises from the combination of its "bottom-up" components - affective compositional effects - and its "top-down" components - affective context [2]. That is, group emotion results from both the combinations of individual level affective factors that group members possess, as well as from group or contextual level factors that define or shape the affective experience of the group. The concept of group emotion has been shown to be reliably recognized by group members and outside raters, both on-site and through video-ratings [3], [4], [5], and has been reliably measured through a variety of statistical techniques. In our application we aim to support emotional awareness as one of the key aspects of the system. 


\section{Technological Description of the Current Application}

The system presented here consists of a mobile phone application and a server-side component. The server links into a database and performs logging and aggregation, as well as communication with the mobile application. There are aggregation rules for each measurable attribute and new aggregation rules and attributes are easy to add. The attributes are identified by name only, and the server-side application searches for a handler method for that particular attribute name. The mobile application is a J2ME MIDlet that targets MIDP 2.0 and CLDC 1.0. It facilitates text-based group communication while also showing a graphical representation of various cues about the group's status.

These attributes are gathered using a combination of self-report questions and various sensors. Sensors are either attached using Bluetooth or are local to the phone. The application does not directly distinguish between different data sources in its communication with the server, so any kind of data source can be added easily. Different phone models support different sets of sensors, and the application can adapt to this. The sensor attachment is implemented with a Bluetooth discovery for known sensor identifiers. The sensor data is periodically transmitted to a server which performs aggregation for each group. Group management is implemented on the server. Each user can belong to several groups. The aggregate values for each group and user are sent to the phone and represented there graphically in a way that depends on the screen size and device capabilities.

The application is a data collection and visualization system implemented on top of a UI library developed to work around the limitations of MIDP2. The library allows for rapid user interface and interaction model development on the limited MIDP2 platform. The application provides a set of predefined visualizations with variable parameters. The different group and individual attributes can easily be mapped at runtime to visualization parameters. This mapping can also be dynamically changed.

\subsection{Information Input to the System}

At the current stage self-report questions about subjective emotional valence and arousal and also about stress level are sent from the server to the users. The frequency of requests can be set from the server and the suitable frequency can be adjusted for different user groups. In the future at least part of these self-report questions are planned to be replaced by data from Bluetooth connected psychophysiological sensors, e.g. skin conductance and pulse from the fingers.

Other types of user input information are status message and instant messaging. Status message is a user typed short description of his or her current work stage or subjective feelings, in a way a profile that is visible to others and permanent until user changes it. Instant messaging allows informal textual communication between group members without the charges and limitations of SMS.

Nearby Bluetooth devices and ambient sound are collected and visualized to provide contextual information. Location data is derived from pre-set Bluetooth beacons; with Symbian phones cell-id can also be accessed. A hierarchical three level model is formed from the cell-id data. In the closest "orbit" are located those who are in the same cell with the user in the center (see Figure 1). The second orbit shows 
those in the same area and the third furthest orbit is a place for those who are not in the same area.

The Bluetooth framework is flexible enough to support essentially any sensor that can communicate over RFCOMM or L2CAP. This enables the use of the system to collect and visualize data not directly accessible from the phone, such as the psychophysiological sensor data mentioned above, as well as more complex external sensors that do processing the phone is not currently capable of (such as voice analysis). The data collection framework and visualization system treats sensor data in exactly the same way as any other attribute, so it is possible to map the attributes to any visualization parameters.

\section{Visualization of Emotional and Contextual Cues}

Visualizing data on mobile phone is still challenging. The screen size and the resolution are quite small and usually the screen orientation is vertical. We have selected an orbit schema for visualizing the group member's data. This way the user can, with a single glance, get information on each other group member. The user is in the center of the orbits and a "planet" represents each other group member. The proximity of other members to the user represents the similarity of emotional ratings or geographic distance.

\subsection{Emotional Information - Sheet}

In the Emotion-sheet self-reported emotional valence, emotional arousal, and stress level are visualized for each group member. The size of the "planet" represents the stress level with larger planet standing for larger amount of stress. Emotional valence is visualized with the color, blue is for negative valence and red for positive valence. The amount of fill in the planet shows the arousal level; low fill is for low arousal level. All these self-report data have been obtained by five-scale questionnaires. The proximity of planets representing users is calculated by forming an average for these three scales together.

\subsection{Contextual Information - Sheet}

In the context sheet the proximity of the planets represents geometric distance between group members, relative to the user placed in the center. The dots around planets show the number of surrounding Bluetooth devices and an anchor stands for proximity to a user's Bluetooth-enabled tabletop PC in the office or a Bluetooth sensor placed in the office room. The fill of the planet and the color of the fill represent ambient sound level and user activity. Status message and detailed information of surrounding Bluetooth devices can be seen from the individual user details (Figure 1, panels E and F). A green person figure represents proximity to a known persons Bluetooth enabled mobile phone. A black mobile phone is for other, unknown persons and a diamond represents any other Bluetooth enabled device.

With the presented visualization approach location, contextual and subjective emotional information can be effectively visualized in a mobile phone. Navigation requires a minimal number of buttons. The Bluetooth and cell-id based approach also makes it possible to access location information without an external GPS receiver, 
although it is possible in the forthcoming versions to include a specific map sheet for visualizing GPS-based location data. Our mobile application is targeted to lessen misunderstandings by providing for a richer interaction between distributed group members. Visibility of other's locations, context and emotions will be enhanced by our system. Enhancing visibility may also lead to more accessibility as people are more aware of the suitable moments to contact friends or co-workers.
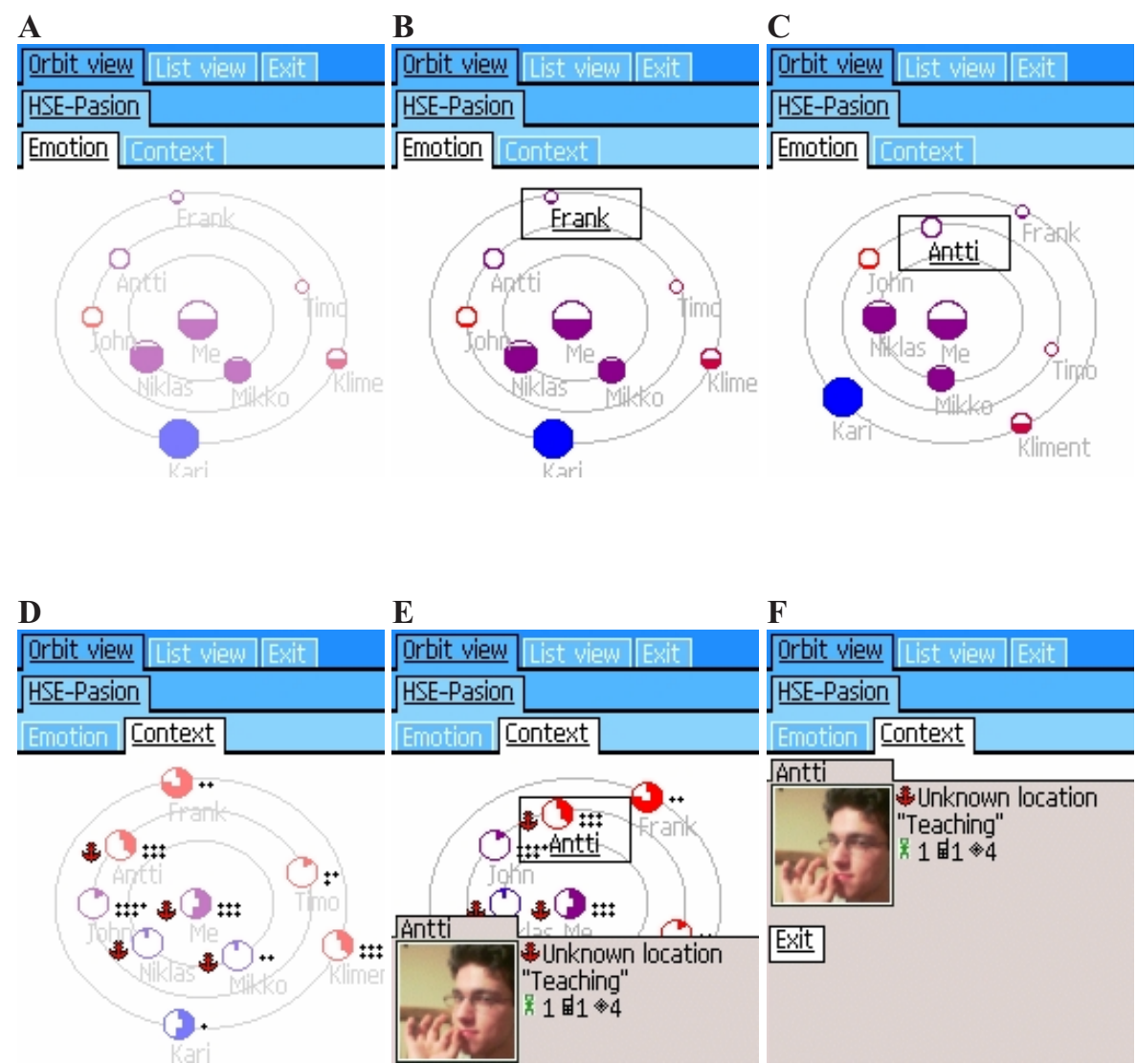

Fig. 1. Navigating in the application and cues for emotional and contextual information. Each group member is visualized as a "planet" in the orbit. First row, sheet for emotional cues: From A view to $\mathrm{B}$ view can be moved by zooming and from $\mathrm{B}$ to $\mathrm{C}$ by clicking previous. Second row, sheet for contextual cues: From $\mathrm{D}$ view to $\mathrm{C}$ view can be moved by zooming and from $\mathrm{E}$ to $\mathrm{F}$ by clicking Action.

We claim that increased socio-emotional awareness is relevant to distributed knowledge work teams and that it may increase efficiency and innovativeness of work. We claim that the mechanism by which this may happen is the increased communication bandwidth and contact frequency in the intimate and non-verbal communication flow within the group enabled by our prototype application. This may 
lead to various individual and group level beneficial effects, such as more efficient emotional regulation, mood management and general emotional awareness, linked to group trust and cohesion, for instance.

The application is at the moment in test use by a small team of knowledge workers. By the end of the year it will be used in large scale trials in four European countries with +100 users in each. In the trials it is possible to collect both qualitative (i.e. interviews, focus groups) as well as quantitative data (i.e. server log data of user performed actions, self-report questionnaires). On the server side it is possible to switch on and off certain cues in the application in order to study the effects of these cues/functions in a more controlled fashion.

Acknowledgements. The work has been conducted within the PASION (Psychologically Augmented Social Interaction Over Networks) Integrated Project, which is funded under the Presence II Initiative in the Future Emerging Technologies within the 6th Framework Program.

\section{References}

1. Herbsleb, J., Grinter, R.: Architectures, Coordination, and Distance: Conway's Law and Beyond. IEEE Software, pp. 63-70 (September/October 1999)

2. Barsade, S.G., Gibson, D.E.: Group emotion: A view from top and bottom. In: Gruenfeld, D., Mannix, B., Neale, M. (eds.) Research on Managing Groups and Teams, pp. 81-102. JAI Press, Stamford (1998)

3. Barsade, S.G.: The Ripple Effect: Emotional Contagion in Groups. In: Working Paper 98. Yale School of Management, Yale University, New Haven, CT (2000)

4. Bartel, C., Saavedra, R.: The collective construction of work group moods. Administrative Science Quarterly 45, 197-231 (2000)

5. Totterdell, P., Kellet, S., Teuchmann, K., Briner, R.B.: Evidence of mood linkage in work groups. Journal of Personality and Social Psychology 74, 1504-1515 (1998) 